\title{
INFLUENCE OF COMPUTED TOMOGRAPHY DETECTOR RESOLUTION ON MEASURED VALUES OF SHAPE AND DIMENSIONAL ACCURACY
}

\author{
Róbert Hrušecký, Ivan Molnár, Augustín Görög \& Dávid Michal
}
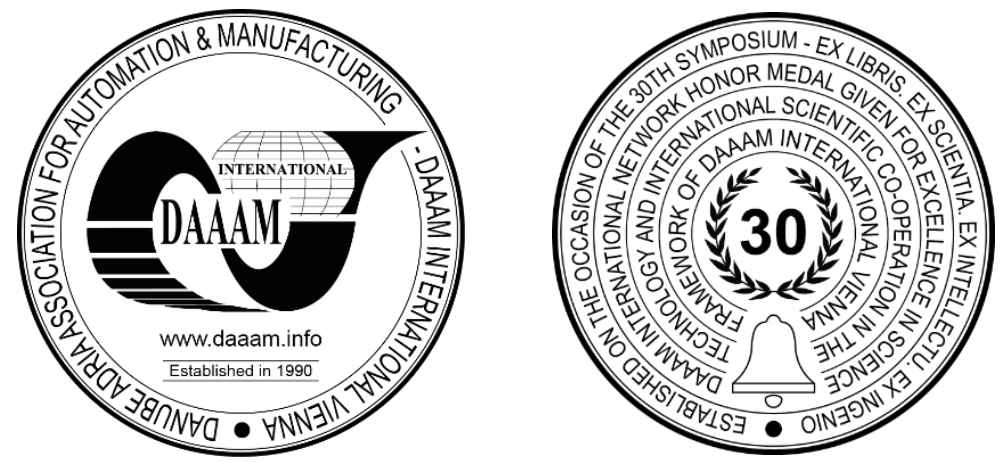

This Publication has to be referred as: Hrusecky, R[obert]; Molnar, I[van]; Gorog, A[ugustin] \& Michal, D[avid] (2019). Influence of Computed Tomography Detector Resolution on Measured Values of Shape and Dimensional Accuracy, Proceedings of the 30th DAAAM International Symposium, pp.0693-0700, B. Katalinic (Ed.), Published by DAAAM International, ISBN 978-3-902734-22-8, ISSN 1726-9679, Vienna, Austria

DOI: $10.2507 / 30$ th.daaam.proceedings.095

\begin{abstract}
Computed Tomography (CT) machines are currently used mainly in applications where it is necessary to determine the various parameters and properties of the internal structure of the measured components. For the most accurate measurements of the external shape and dimensions of the components, Coordinate Measuring Machines (CMM) are used. The article describes the effect of settings of the resolution of the CT detector on the accuracy of the measurement results of the shape and dimensions of the component produced by the Material Jetting Process.
\end{abstract}

Keywords: Computed Tomography; Coordinate Measuring Machine; Material Jetting Process; CT detector.

\section{Introduction}

Although CT has been developed and used in medical and material science for several decades, its application to dimensional metrology is still evolving and improving. Compared to conventional component scanning methods, computed tomography brings many options that have been lacking in digitization and quality control. [1]. A significant advantage is obtaining information in areas that are inaccessible to conventional 3D coordinate measurement technology (whether camera or laser). At present, very complex injection molding or casting parts are present in multi-part molds, which often cannot be controlled by another method like CT. By X-ray scanning of a real part during rotation, we get its virtual model, which can be freely rotated, explored in sections, or subjected to further analysis [2].

Currently, AM technology is increasingly used in various industries around the world. From the beginning of this technology, from the initial production of prototypes in the development phase of future products, we are now coming to the design and manufacture of final products for use in practice. This is the result of the ever-evolving field of additive technologies, where improvements are made and the efficiency of the production process, parameters of production equipment and the modification and development of new types of component building materials are improved. Thanks to this, AM is becoming increasingly used and expanding its application in many industries [3].

Paper deals with the influence of setting CT detector resolution on the accuracy of geometrical characteristics of component shape and dimensions. The component was produced using the Material Jetting additive process. As a result, 
it will be possible to see both the effect of different detector settings and the precision of production on a Material Jetting technology, which is increasingly being used to produce precision plastic components. Some CT devices have different detector parameters. At present, devices with a $3 \mathrm{~K}$ detector (3008 x 2512 pixels) have been produced which means smaller voxel and better accuracy. When the detector is set to a higher resolution, the measurement time and data are increased several times. The main aim of this paper is to find out how much influence the detector resolution has on measurement accuracy.

\section{Additive Manufacturing - Material Jetting Process}

Additive Manufacturing (AM) is the formalized term for what used to be called Rapid Prototyping and what is popularly called 3D Printing. The term Rapid Prototyping (RP) is used in a variety of industries to describe a process for rapidly creating a system or part representation before final release or commercialization [3].

The AM is about the latter technologies, first developed for prototyping, but now used for many more purposes. Users of RP technology have come to realize that this term is inadequate and in particular does not effectively describe more recent applications of the technology. Improvements in the quality of the output from these machines have meant that there is often a much closer link to the final product. The basic principle of this technology is that a model, initially generated using a three-dimensional Computer-Aided Design (CAD) system, can be fabricated directly without the need for process planning [4].

The AM technology certainly significantly simplifies the process of producing complex 3D objects directly from CAD data. Other manufacturing processes require a careful and detailed analysis of the part geometry to determine things like the order in which different features can be fabricated, what tools and processes must be used, and what additional fixtures may be required to complete the part.

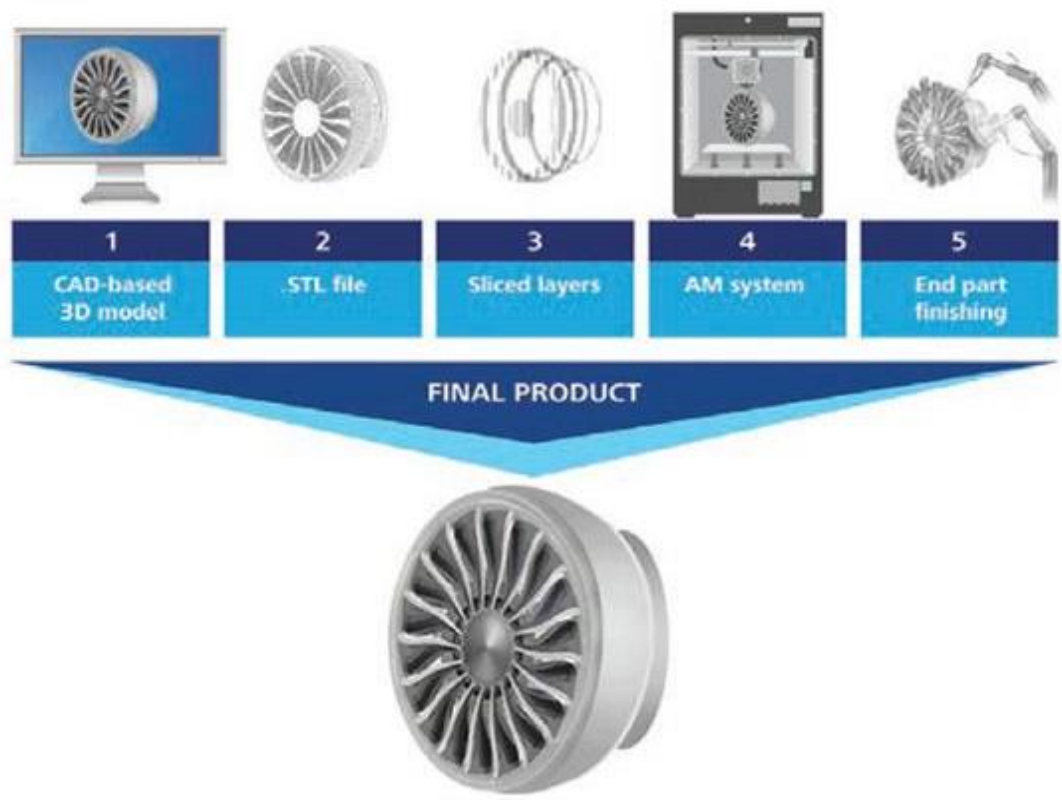

Fig. 1. The basic process of AM

The AM technology certainly significantly simplifies the process of producing complex 3D objects directly from CAD data. Other manufacturing processes require a careful and detailed analysis of the part geometry to determine things like the order in which different features can be fabricated, what tools and processes must be used, and what additional fixtures may be required to complete the part. The key to how AM works is that parts are made by adding material in layers; each layer is a thin cross-section of the part derived from the original CAD data. Obviously in the physical world, each layer must have a finite thickness to it and so the resulting part will be an approximation of the original data [5].

All commercialized AM machines to date use a layer-based approach, and the major ways that they differ are in the materials that can be used, how the layers are created, and how the layers are bonded to each other. Such differences will determine factors like the accuracy of the final part plus its material properties and mechanical properties. They will also determine factors like how quickly the part can be made, how much post-processing is required, the size of the AM machine used, and the overall cost of the machine and process.

The plastic component mentioned in this article was produced by Material Jetting or MultiJet Printing (MJP) additive process. The MJP is an inkjet printing process that uses printhead technology to deposit either photocurable plastic resin or casting wax materials layer by layer. MJP is used to build parts, patterns and molds with fine feature detail to address a wide range of applications [6]. 


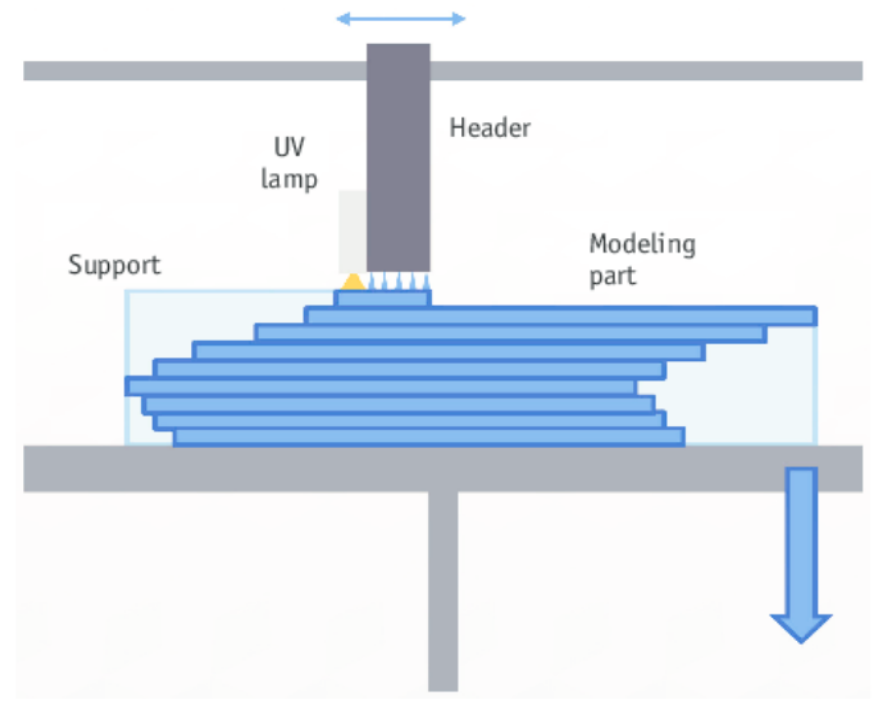

Fig. 2. The basic principle of Material Jetting process

These high-resolution printers are economical to own and operate and use a separate, meltable or dissolvable support material to make post-processing a breeze. Another big benefit is that removing support material is virtually a hands-free operation and allows even the most delicate features and complex internal cavities to be thoroughly cleaned without damage [3]. Material Jetting or also called Ink- jet or droplet printing technology has rapidly developed in recent years. Improvements in resolution and reduction in costs has meant that high-resolution printing, often with multiple colors [7].

In the context of this study, Material Jetting process was chosen due to it its high accuracy, speed of production and hence wide application possibilities.

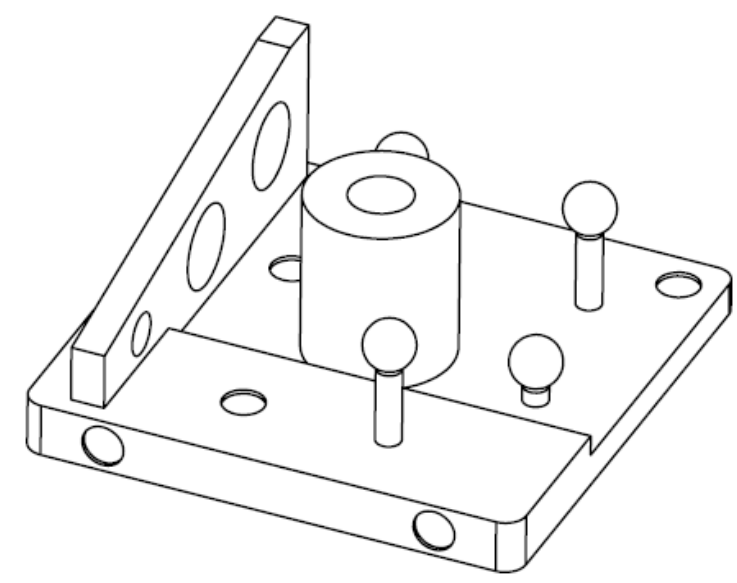

Fig. 3. Component produced by Material Jetting Process

\section{CMM and CT Measurements}

Coordinate measurement technology is one of the most accurate data acquisition technologies. It is a computercontrolled device that has a complex measurement system and is capable of measuring in space or in the plane of a given coordinate system. The coordinate measuring machine works with at least two coordinate systems: the coordinate system of the measured object and the coordinate system of the machine. It allows maximum accuracy in high-speed scanning. Automatically sets the maximum scanning speed for scanning with guaranteed accuracy. ZEISS PRISMO uses a computer-controlled correction of all dynamic effects on the measuring machine. This optimizes high-speed scanning accuracy. The component was measured on a ZEISS Prismo Ultra where the maximum permissible measurement error $\mathrm{MPEE}=0.5+\mathrm{L} / 500 \mu \mathrm{m}$. Measurements on CMM were performed in laboratory conditions 10 times and the results were considered as reference values [8]. 


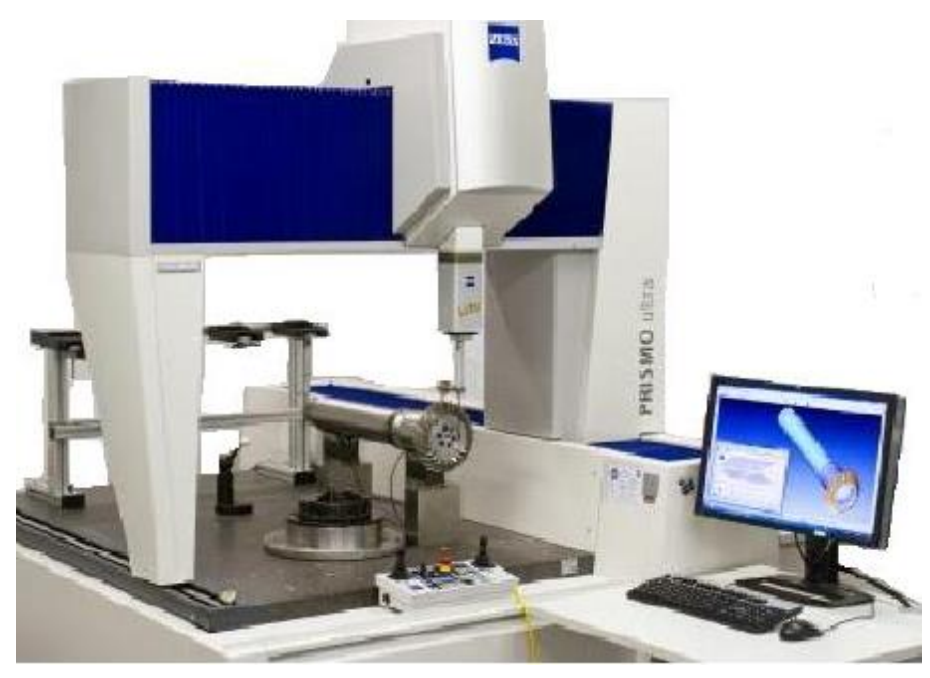

Fig. 4. CMM PRISMO ultra in laboratory on MTF STU

At present, dimensional CT metrology is the only technology capable of measuring both internal and external component geometry without the need for cutting and destruction. At present, very complex injection molding or casting parts are present in multi-part molds, which often cannot be controlled by another method [9]. Dimensional CT metrology often requires high radiation energy because the demand for measuring larger and thicker workpieces made from more absorbent materials (e.g., metals) is increasing. In addition, dimensional quality control requires high spatial resolution and accuracy due to measurement uncertainties [2].

As computer tomography is a new technology in metrology, there are not yet comprehensive objective standards for measuring uncertainty. Carl Zeiss has developed a reference standard consisting of 27 ruby balls mounted on carbon rods to verify their tomographs. For the individual pairs of spheres, the distances of their centers are measured, thereby checking the different length dimensions in different directions. The measurement is performed 50 times. Neither of the deviations of the measured lengths may exceed $(9+\mathrm{L} / 50) \mu \mathrm{m}$. Of course, other versions of reference standards can also be used to verify [8].

$\mathrm{X}$-rays are generated when the charged particles are accelerated or when the electron charge changes in the atom. The thermal electrons are excited on the glowing tungsten fiber and are accelerated by the electric field towards the target. In industrial X-ray tomographs, a micro-focal radiation source is used compared to medical CTs. At this source, the resulting thermal electron beam is focused by magnetic lenses. This makes it possible to select the size of the footprint on the target. The electrons flowing through the tube are accelerated and thus increase their energy, thereby increasing the X-ray intensity. After impacting the detector, the intensity of the output radiation intensity is less than the input intensity [10].

On the Figure 5 is a scanning and data retrieval procedure from computer tomography. The source generates $\mathrm{X}(\mathrm{X}$ rays) rays. Since X-rays spread through the workpiece material, they are weakened due to absorption or scattering. The amount of attenuation is determined by the length passed in the absorbent material, the composition of the material and its density and the X-ray energy [11]. The attenuation is measured by capturing the remaining X-rays that pass through the workpiece using an X-ray detector, resulting in a 2D gray image [12]. The mathematical reconstruction of these projected images leads to a 3D voxel model. The next steps concern the post-processing of voxel data, including workpiece edge detection (segmentation), and subsequent measurement and quality control [13].

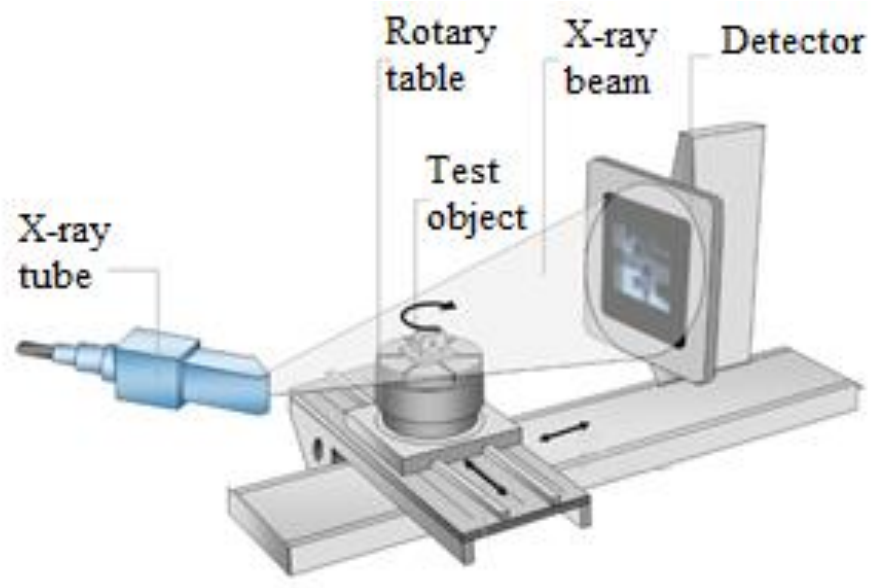

Fig. 5. Basic design of industrial tomographs 
The aim of the experimental work was to scan the component for the same parameters only when changing the detector resolution from $1 \mathrm{~K}(1024 x 1024$ pixels $)$ to $2 \mathrm{~K}$ (2048x2048pixels). Scanning parameters are listed in Table 1 . We also change the number of frames and voxel size when resizing. The term voxel (volumetric element) was created as an analogue of a two-dimensional pixel. Indicates the smallest element in three-dimensional discrete space. When placing these elements in a three-dimensional regular grid, we call this grid a voxel grid. As with pixels, the voxel does not implicitly recognize its position in the voxel grid, but it is accessed by coordinates $(\mathrm{x}, \mathrm{y}, \mathrm{z})$, similar to the pixel value in a two-dimensional grid. Voxel can be imagined as a filled block, which has a constant value throughout its volume. Voxels are used for fully three-dimensional representation of objects and allow to capture any surface and terrain. A major disadvantage is their data intensity and slow display time [12].

\begin{tabular}{|c|c|c|}
\hline & $\begin{array}{c}\mathbf{1 K} \\
\mathbf{( 1 0 2 4 x 1 0 2 4} \text { pixel) }\end{array}$ & $\begin{array}{c}\mathbf{2 K} \\
\mathbf{( 2 0 4 8 \times 2 0 4 8} \text { pixel })\end{array}$ \\
\hline Voltage $[\mathrm{kV}]$ & 170 & 170 \\
\hline Current $[\mathrm{uA}]$ & 700 & 700 \\
\hline Integration time $[\mathrm{ms}]$ & 500 & 500 \\
\hline Gain & $8.0 \mathrm{x}$ & $8.0 \mathrm{x}$ \\
\hline Number of projections & 1050 & 2050 \\
\hline Spot size[um] & 85 & 85 \\
\hline Voxel size $[\mathrm{um}]$ & 146 & 74 \\
\hline
\end{tabular}

Table 1. Scanning parameters

Measurements were made on a ZEISS Metrotom 1500 computer tomography. The CT device has a $2 \mathrm{~K}$ detector. The scanning parameters are set depending on the size of the measured component, the transmissibility of the X-rays by the sensed material and the purpose of the data to be taken before the actual scanning. It is necessary to position the component so that the image in the field of view of the detector occupies the largest possible area during rotation around the vertical axis.

\begin{tabular}{|l|c|c|c|}
\hline Characteristic & CMM [mm, $^{\circ}$ ] & CT 1K [mm, ${ }^{\circ}$ ] & CT 2K [mm, ${ }^{\circ}$ ] \\
\hline 01_Dia. 30 & 29.9907 & 29.9583 & 29.9975 \\
\hline 02_Dia. 15 & 14.9726 & 14.9778 & 15.0055 \\
\hline 03_Coax. 01 & 0.0183 & 0.0182 & 0.0214 \\
\hline 04_Dia. 14 & 13.9990 & 13.9605 & 13.9843 \\
\hline 05_Dia. 15 & 14.9909 & 14.9618 & 14.9802 \\
\hline 06_Dia. 12 & 11.9946 & 11.9653 & 11.9857 \\
\hline 07_Dia. 12 & 11.9945 & 11.9667 & 11.9822 \\
\hline 08_Pos. 1 & 0.2660 & 0.3068 & 0.2869 \\
\hline 09_Pos. 2 & 0.0878 & 0.1214 & 0.1260 \\
\hline 10_Pos. 3 & 0.3521 & 0.3901 & 0.3709 \\
\hline 11_Pos. 4 & 0.3043 & 0.3399 & 0.3123 \\
\hline 12_Angle 12 & 11.8339 & 11.8327 & 11.8154 \\
\hline 13_Dia. 20 & 19.9981 & 20.0265 & 20.0120 \\
\hline 14_Dia. 10 & 9.9780 & 10.0097 & 10.0066 \\
\hline 15_Flatness & 0.2873 & 0.2920 & 0.2736 \\
\hline 16_Paral & 0.2320 & 0.2575 & 0.2285 \\
\hline 17_Perpen & 0.1691 & 0.1851 & 0.1837 \\
\hline 18_Dis.100_X & 100.0202 & 100.0018 & 100.0492 \\
\hline 19_Dis.100_Y & 100.0310 & 100.0056 & 100,0570 \\
\hline
\end{tabular}

Table 2. Measurement results from coordinate measuring technique and computer tomography on the measured component 
Before measurement, the number of positions per revolution is also set, that is, the number of images to be saved for reconstruction. The device was heated and calibrated by calibration standards to the geometric and axial calibrations that were clamped on the turntable prior to measurement. Calibration ensures dimensional accuracy of the measuring system. It has been performed according to the manufacturer's recommended procedure. It consisted of recording images at different distances, positions, and orientations of the calibration standard relative to the emitter and detector. The measured component has been designed to measure the basic dimensional and shape characteristics of the component. The part was printed on a 3D Systems ProJet 2500 device where the printing accuracy is $0.025-0.05 \mathrm{~mm}$ to $25.4 \mathrm{~mm}$. VisiJet M2R-WT [6].

The experimental work was carried out in the Center of Excellence of Five-Axis Machining at the Faculty of Materials Science and Technology in Trnava, Slovak Technical University in Bratislava, where ZEISS Metrotom 1500 is located and used in educational and research processes. For Metrotom 1500 in its measuring range, the maximum permissible error for MPEE $=(9+\mathrm{L} / 50) \mu \mathrm{m}$ is given by the manufacturer [8].

As mentioned above, two detector settings were used in the experiment. The component was measured on a ZEISS Prismo Ultra where the maximum permissible measurement error MPEE $=(0.5+\mathrm{L} / 500) \mu \mathrm{m}[8]$. Measurements on CMM were performed in laboratory conditions 10 times and the results were considered as reference values. The specific program for the component measurement was created in software ZEISS Calypso and it was used for CMM and CT scan. The CT scanner was scanned 5 times with $2 \mathrm{~K}$ resolution and $1 \mathrm{~K}$ resolution. The maximum permissible measurement error of the MPEE device was not included in the results.

The author of paper monitored measurements of dimensional and geometrical characteristics. Research included observation of influence parameter geometrical magnification on measurement results obtained with usage of computed tomography. Authors changed the distance between x ray tube and measured object, and it is Geometrical magnification. The results showed deviations from reference values [14].

On the Figure 6 is a graphical representation of dimensional values such as diameters and distances. These values are the result of differences in the arithmetic mean of the reference values obtained from the coordinate measurement technique and the arithmetic means from the computed tomography at full resolution of $2 \mathrm{~K}$ or $1 \mathrm{~K}$ half resolution. In the graphical representation, we can see that at full resolution the values are closer to zero. That is, the full resolution of the detector, or $2 \mathrm{~K}$, is more suitable for use for more accurate measurements. Only in one case did the full resolution have greater differences from the reference than half $1 \mathrm{~K}$.

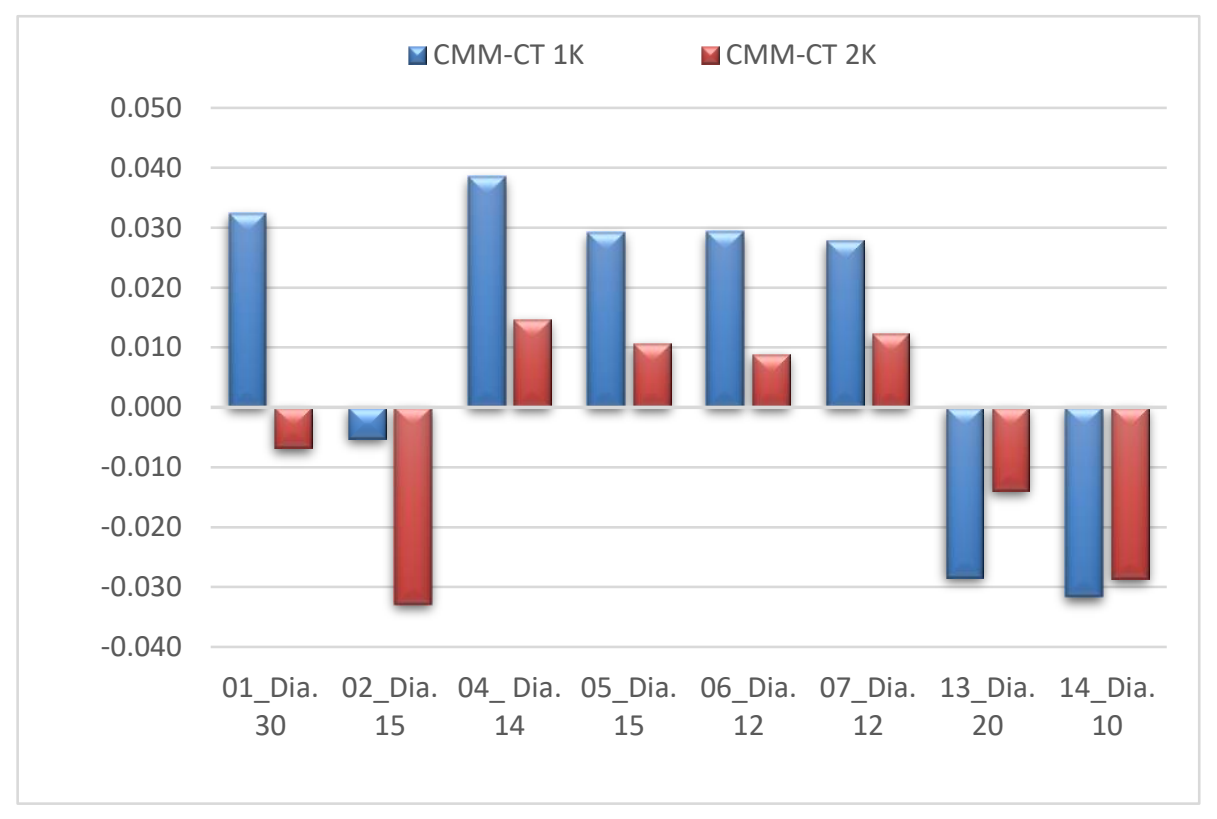

Fig. 6. Graphical representation of diameter difference from reference value [mm]

On the Figure 7 is a graphical representation of shape characteristics such as flatness, perpendicularity, alignment. These values are the result of differences in the arithmetic mean of the reference values obtained from the surrogate measurement technique and the arithmetic means from the computed tomography at full $2 \mathrm{~K}$ resolution or half $1 \mathrm{~K}$. Values are not as significant as in Figure 6. Values show a greater difference between the reference value and the value obtained from computer tomography. 


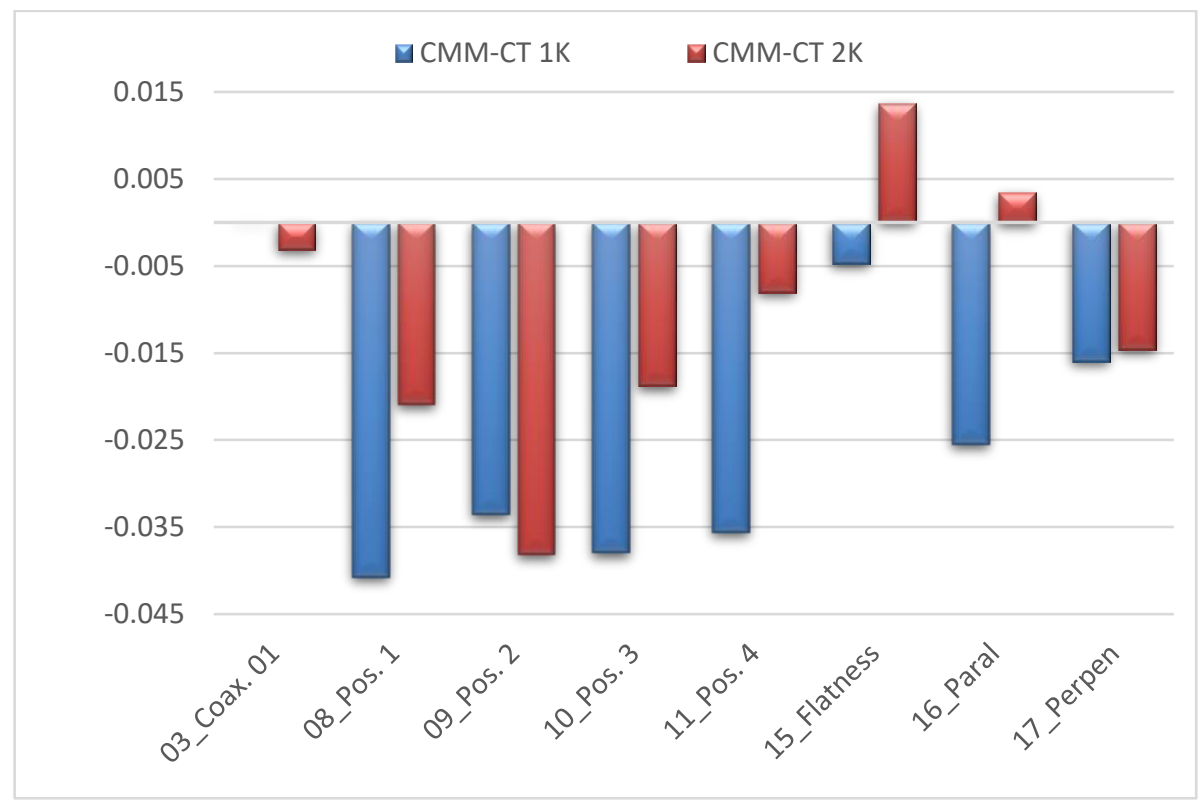

Fig. 7. Graphical representation of the difference between the shape values and the reference value

\section{Conclusion}

The measured values indicate that the detector setting had affects the accuracy of dimensional characteristics such as diameters, angle and distance. When measured outer diameter on a CT device, it was observed that the diameter was larger than the reference value and inner diameter measurements, the diameter was less than the reference right. Measured values at full detector resolution they were closer to the measured values from the coordinate measuring technique, which we consider the reference value. Measurement results at different detector settings on shape characteristics are not so unambiguous. Evaluation of shape deviations showed differences from the reference values results were unpredictable. Position measurements showed large differences over 5 measurements. The advantage of half the resolution $1 \mathrm{~K}$ of the detector over the full resolution $2 \mathrm{~K}$ is the measurement time, which is almost half. Scanning the part at half the resolution of the detector took 17 minutes and at full resolution the measurement time took 29 minutes.

This article discusses changing the resolution to change the number of images and voxel size. Another research could be about the influence of the voxel size or the number of images on the accuracy of this component, which is made using MJP technology.

Purpose of the carried out experiment was to determine the most optimal set of conditions for future measurement of solid turning tools with prepared edges.

\section{Acknowledgments}

This work was supported by the Scientific Grant Agency of the Slovak Republic under the grant no. 1/0097/17 and the plastic component described in the article was manufactured and supplied by Advanced Engineering company in partnership with Elvira company.

\section{References}

[1] Kat'uch, P., Kovač, J., Dovica, M. (2010). Analysis of mechatronic systems using computer tomography. 17th Symposium IMEKO TC 4, 3rd Symposium IMEKO TC 19 and 15th IWADC Workshop Instrumentation for the ICT Era, Kosice, Slovakia.

[2] Jansson, A., Zekavat, A.R., Pejryd L. Measurement of internal features in additive manufactured components by the use of computed tomography. Digital Industrial Radiology and Computed Tomography (DIR 2015) Ghent (2015)

[3] Gibson, I., Rosen, D., Stucker, B. (2015). Additive manufacturing technologies (Second Edition). Springer New York Heidelberg Dordrecht London, 2015, 498 pages, ISBN: 978-1-4939-2112-6.

[4] Boubolous, M. A. (2010). CAD - CAM \& rapid prototyping application evaluation. 174 s. ISBN 978-87-7681-6766.

[5] Nguyen, H., Vai, M. (2010). Rapid prototyping technology. Lincoln laboratory journal, Volume 18, Number 2.

[6] 3DSystems, ProJet MJP 2500/2500 PLUS, (2019) Retrieved from https://www.3dsystems.com/3d-printers/projetmjp-2500-series

[7] Sturm, L. D., Albakri, M. I., Tarazaga, P. A., Williams, Ch. B. (2019). In situ monitoring of material jetting additive manufacturing process via impedancebased measurements. Additive Manufacturing, 28 (2019), 456-463. 
[8] ZEISS Systems, (2016) Retrieved from https://www.zeiss.com/metrology/products/systems.html

[9] Gapinski, B.; Wieczorowski, M.; Marciniak-Podsadna, L.; Dybala, B. \& Ziolkowski, G. (2013). Comparison of Different Method of Measurement Geometry using CMM, Optical Scanner and Computed Tomography 3D, Procedia Engineering 69, 24th DAAAM International Symposium on Intelligent Manufacturing and Automation, Zadar, Katalinic, B. (Ed.), pp.255-262, Elsevier, DOI:10.1016/j.proeng.2014.02.230

[10] Baruchel, J. (2000). X-ray tomography in material science. Paris: Hermes Science. ISBN: 2746201151.

[11] Kruth, J.P.; Bartscher, M.; Carmignato, S.; Schmidt, R.; De Chiffre, L. \& Weckenmann A. (2011). Computed tomography for dimensional metrology, CIRP Annals - Manufacturing Technology Vol. 60, No. 2, 2011, pp.821842, ISSN 0007-8506; DOI 10.1016/j.cirp.2011.05.006

[12] Maret, D., Telmon, N., Peters, A.,Lepage, B., Treil, J.,Peyre, A. 2012. Effect of voxel size on the accuracy of 3D reconstructions with cone beam CT, Dentomaxillofac Radiol. 2012 Dec; 41(8): 649-655.

[13] Carmignato, S., Wim Dewulf, Richard Leach. (2018) - Industrial X - Ray Computed Tomography, Springer, ISBN 978-3-319-59571-9, (2018). DOI: https://doi.org/10.1007/978-3-319-59573-3

[14] Horvatic, A., Runje, B. \& Butkovic, D. (2016). Influence of Geometrical Magnification on Computed Tomography Dimensional Measurements, Proceedings of the 27th DAAAM International Symposium, pp.0615-0622, B. Katalinic (Ed.), Published by DAAAM International, ISBN 978-3-902734-08-2, ISSN 1726-9679, Vienna, Austria DOI: $10.2507 / 27$ th.daaam.proceedings.090. 